\title{
Earthworm Castings as a Substrate Amendment for Chrysanthemum Production
}

\author{
Pablo R. Hidalgo ${ }^{1}$ and Richard L. Harkess ${ }^{2}$ \\ Department of Plant and Soil Sciences, 117 Dorman Hall, Box 9555, Mississippi \\ State University, Mississippi State, MS 39762
}

\section{Additional index words. Dendranthema $\times$ grandiflora, vermicompost, animal waste, horse manure, cattle manure, sheep manure, flower development}

\begin{abstract}
Earthworm castings (vermicompost) were evaluated as a substrate amendment for chrysanthemum [Dendranthema xgrandiflora (Ramat.) Kitam.] 'Miramar' production. Vermicompost produced from sheep, cattle, and horse manures were mixed at different ratios with 70 peatmoss : 30 perlite (v/v) to create 12 substrates. The 70 peatmoss : 30 perlite mix at $100 \%$ and Sunshine ${ }^{\circledR}$ Mix 1 were used as control substrates. The bulk density, percentage of pore space, and water holding capacity increased as vermicompost content increased while the percentage of air space decreased. At $100 \%$ vermicompost, water holding capacity and bulk density were greatest in vermicompost from sheep manure. Plants grown in mixtures of $\mathbf{5 0 \%}$ vermicompost from sheep had a greater growth index at harvest, foliar area, number of flowers per pot, and dry weight and fewer days for flower development than plants grown in other substrates. Vermicompost from sheep manure added at $50 \%$ by volume was most effective as a substrate amendment for chrysanthemum production.
\end{abstract}

Chrysanthemums are second only to poinsettias in crop value (Miller, 2000). Chrysanthemums are largely leafy plants and naturally heavy feeders during their growing cycle. Most producers grow mums in soilless mixes, which include peat, pearlite, and vermiculite (Wang and Pokorny, 1989). Of these, sphagnum peatmoss has been the dominant bulk material in substrates for potted plants for more than 30 years (Hansen et al., 1993). For several decades, peat has been reported to be expensive, variable, and increasingly unavailable (Sanderson and Martin, 1974). In addition, since the beginning of the 1990 s, environmentalists have been pressing the horticulture industry to avoid its heavy use of peat as a growing substrate claiming that unique, lowland wetlands are being destroyed by the peat extraction procedure (Neal, 1991).

Vermicompost is an alternative substrate that has been available for several years. Vermicomposting is the processing of organic

Received for publication 12 July 2001. Accepted for publication $25 \mathrm{Feb}$. 2002. This paper is a portion of the dissertation submitted by P. Hidalgo in partial fulfillment of the requirements for the PhD degree in Horticulture at Mississippi State Univ. We thank Yoder Bros. (Barberton, Ohio) for the donation of the plant material used in this research. Contribution of the Mississippi Agricultural and Forestry Experiment Station journal article no. J9885. Mention of trademark or proprietary product does not constitute a guarantee or warranty of the product and does not imply its approval to the exclusion of other products that may also be suitable.

${ }^{1}$ Graduate Research Assistant. E-mail address: biruaca@mail.usa.com

2Associate Professor. To whom reprint requests should be addressed. E-mail address: harkessr@ ra.msstate.edu wastes with earthworms. During this process, elements like $\mathrm{N}, \mathrm{P}, \mathrm{K}$, and $\mathrm{Ca}$ present in the waste are released and converted, through microbial activity, into forms more soluble and available to plants than those present in the original waste (Edwards, personal communication, 1998). Vermicompost chemical composition depends not only on the degree the worms have been working on the wastes but also on the composition of the initial organic waste (Handreck, 1986). This process has been used extensively to transform agricultural waste into organic fertilizer. Orozco et al. (1996) allowed earthworms to work on coffee pulp wastes and found that vermicomposting increased available $\mathrm{P}, \mathrm{Ca}$, and $\mathrm{Mg}$, decreased $\mathrm{K}$, but had no effect on $\mathrm{C}$ and $\mathrm{N}$ availability from the initial material.

Earthworm compost has potential for horticultural use, particularly as a potted-plant substrate (Buchanan et al., 1988). Tomar et al. (1998) grew Daucus carota L. in pots containing soil alone or with the addition of farmyard manure, or vermicompost and obtained the greatest yield when vermicompost was used. Kalembasa et al. (1998) evaluated the effect of different sources of $\mathrm{N}$ (farmyard manure, ammonium nitrate, and vermicompost produced from waste activated sludge and from meat processing factory waste) and noted that Raphanus sativus L. (Radicula Group) and Capsicum annuum L. var. annuum (Grossum Group) cultivated in pots produced greater yields when vermicompost was present. Ammonium nitrate also increased $C$. annuum yields but decreased $R$. sativus yields.

Vermicompost produced from the organic fraction of municipal solid waste was evaluated by Alves and Passoni (1997) as a component of potting substrate for Licania tomentosa
Aubl. seedling production. Both seed germination and plant growth increased with vermicompost use. However, in studies conducted by Donald and Visser (1989) survival and growth of Acacia mearnsii De Wildeman. were reduced by use of vermicompost and Eucalyptus grandis W. Hill ex Maid. grew equally well in pure pine bark and in the mixture of 1 pine bark : 1 vermicompost. Survival of Pinus patula Schiede ex Schldl. \& Cham. was not affected by vermicompost, but growth was reduced.

In the present study, earthworm castings produced from sheep, cattle, and horse manures were combined at different ratios with a peatmoss base substrate to create 12 substrates. The objectives were to: 1 ) determine if vermicompost is suitable for chrysanthemum production; 2) which animal waste results in the most suitable vermicompost; and 3) what percentage of vermicompost (by volume) in the substrate results in the best chrysanthemum growth.

\section{Materials and Methods}

Castings produced using the red or tiger worm, Eisenia fetida Sav., feeding on Ovine aries L. (sheep), Bos taurus L. (cattle), or Equus caballus L. (horse) manure were used to create the different substrates evaluated in this experiment. Horse and $75 \%$ of the cattle manure were collected from grazing animals feeding on grass, and were vermicomposted without any pretreatment. Sheep and $25 \%$ of the cattle manure, collected from stabled animals feeding on concentrated feed supplements and hay, were leached with water twice, left for $4 \mathrm{~d}$ and leached again three times to reduce the amount of salt and ammonia present (Edwards, 1988). While cattle and horse manures used in this experiment had an average of 1.25 and $0.90 \mathrm{dS} \cdot \mathrm{m}^{-1}$ electrical conductivity $(E C)$, respectively, sheep manure had $\approx 13.0$ $\mathrm{dS} \cdot \mathrm{m}^{-1}$ before leaching.

Five liters of manure and $170 \mathrm{~g}$ of mostly adult earthworms ( $\approx 425$ individuals) were placed in each $6897-\mathrm{cm}^{3}$ plastic container. The containers were placed in $60 \%$ shade to ensure optimum temperature and light for earthworm survival and growth (Edwards, 1988). Each different manure was replicated 16 times for a total of 48 plastic containers used in the production of castings. Eisenia fetida are reported to consume their body weight in organic wastes on a daily basis and were separated from the castings after 1 month (Riggle and Holmes, 1994). To remain consistent in treatment, castings from horse, cattle, and sheep manure were separated from the worms after the same length of time. Due to differences in digestion between horse, cattle, and sheep, more undigested cellulose was present in the horse manure before being fed to the worms.

The 16 replications of each casting were combined then air-dried and passed through a 6 -mm sieve to harvest $\approx 90 \%$ of the castings. Castings were pooled per type of manure. Twelve treatment substrates were obtained by mixing 70 peatmoss : 30 perlite $(\mathrm{v} / \mathrm{v})$ with castings from each of the three animal manures 
at $100 \%, 75 \%, 50 \%$, or $25 \%$. Peatmoss : perlite $(70: 30 \mathrm{v} / \mathrm{v})$ with no added castings and Sunshine Mix 1 (SunGro Horticulture, Bellevue, Wash.) represented treatments 13 and 14 , respectively, and were used as controls.

Four rooted cuttings of Dendranthema xgrandiflora 'Miramar' (Yoder Bros., Barberton, Ohio) were transplanted per 1-L (15 cm diameter) plastic pot on 19 Feb. 1999 and grown in a double-layer polyethylenecovered greenhouse. Night interruption lighting from 2200 to $0200 \mathrm{HR}$ was used until 4 Mar. The plants were irrigated as needed. The plants were pinched on 3 Mar., removing $\approx 2.5 \mathrm{~cm}$ of new growth. All plants in the experiment were fertilized using Peter's $15 \mathrm{~N}$ 4.3P-24.9K Pot Mum Special (The Scotts Co., Marysville, Ohio) at $300 \mathrm{mg} \cdot \mathrm{L}^{-1} \mathrm{~N}$ by fertigation, except weekends when clear water was applied. Plants were covered with black cloth from 17:00 to 08:00 HR starting on 26 Mar. until flower color development on 11 Apr.

Bulk density, percent pore space, percent air space, water holding capacity, EC, and $\mathrm{pH}$ of the substrates were determined on three samples of each treatment substrate at the beginning of the experiment. Single samples of $100 \%$ castings and the control substrates were delivered to the Mississippi State Univ. Soil Testing Laboratory (Mississippi State, Miss.) to determine macro- and micronutrient content (Cox, 2001).

Every 2 weeks, leachate samples were collected from each pot $1 \mathrm{~h}$ after irrigation using the Virginia Tech pour-through technique (Wright, 1986; Wright et al., 1990). Leachate $\mathrm{pH}$ and $\mathrm{EC}$ were measured using an Accumet Basic pH Meter (Fisher Scientific, Springfield, N.J.) and a YSI model 35 Conductance Meter (Yellow Springs Instruments, Yellow Springs, Ohio), respectively.

At 4 and 10 weeks after planting ( 2 and 8 weeks after pinching), height (h) was measured for each pot from the substrate surface to the shoot apex of the tallest branch. Canopy width (w1) was measured across the side that appeared to be widest, the plant was turned $90^{\circ}$, and a second canopy width measurement (w2) was taken. Using these measurements, the growth index was calculated as:

Growth Index $=\pi \cdot\{[(w 1+w 2) / 2] / 2\}^{2} \cdot h$ (e.g., a volume estimate).

Table 1. Total elemental content of soilless substrates and vermicomposts from three manure types.

\begin{tabular}{|c|c|c|c|c|c|c|c|c|c|c|}
\hline Substrates & $\mathrm{N}$ & $\mathrm{P}$ & $\mathrm{K}$ & $\mathrm{Ca}$ & $\mathrm{Mg}$ & $S$ & $\mathrm{Fe}$ & $\mathrm{Mn}$ & $\mathrm{Zn}$ & $\mathrm{Cu}$ \\
\hline & -- & -- & $\overline{m g} \cdot \mathrm{g}^{-1}$ & -- & -- & -- & $-\mu \mathrm{g} \cdot g$ & -- & - & \\
\hline Sheep & 35.2 & 13.7 & 12.3 & 30.2 & 7.2 & 1000 & 1711 & 314 & 215 & 10 \\
\hline Cattle ca & 5.4 & 14.3 & 7.4 & 31.6 & 11.1 & 1500 & 1285 & 380 & 337 & 40 \\
\hline Horse castings & 39.1 & 9.5 & 8.3 & 18.5 & 5.6 & 860 & 1672 & 245 & 204 & 12 \\
\hline 70 peat : & 14.2 & 0.1 & 0.1 & 2.5 & 0.9 & $\mathrm{n} / \mathrm{d}^{2}$ & 66 & 22 & 78 & 2 \\
\hline Sunshine Mix 1 & 16.5 & 0.7 & 1.5 & 25.9 & 7.7 & $\mathrm{n} / \mathrm{d}$ & 774 & 58 & 39 & 7 \\
\hline
\end{tabular}

${ }^{2}$ Not determined.

At harvest, leaf area, days to harvest, flower number, substrate depth, and shoot dry weight were determined. Plants were harvested when at least 5 flowers in the pot showed petals at least $3 \mathrm{~mm}$ long. Total foliar area was determined using a portable area meter (model LI-3000; LI-COR, Lincoln, Nebr.). Substrate depth was measured from the bottom of the pot to the surface of the substrate. Shoot dry weight was measured per pot on all aboveground growth dried at $60^{\circ} \mathrm{C}$ until dry.

The experiment was arranged in a completely randomized design using 14 treatments and eight single pot replications. Data were analyzed using contrast procedures to determine differences between vermicompost concentrations within compost type. Comparisons between types of compost at each concentration were separated using Fischer's protected least significant difference (LSD) means separation test with $P=0.05$ (Statistical Analysis Software, SAS Institute, Cary, N.C.).

\section{Results and Discussion}

Worm castings appeared to have higher macro and micronutrient content than the controls (Table 1) with the exception of $\mathrm{Ca}$ and Mg where Sunshine ${ }^{\circledR}$ Mix 1 had values greater than the casting from horse manure. The amount of each nutrient was proportionally reduced as the amount of peatmoss increased in the mixture for the three different castings evaluated (data not shown).

Within casting type, bulk density increased as the peat content decreased (Table 2). Sheep castings had greater bulk density than cattle or horse castings. Both controls had lower bulk density than the substrates with castings. With increasing castings content, pore space decreased before increasing as casting content approached $100 \%$. Sheep castings had the least pore space compared to the cattle and horse castings. Percentage of air space was greatest when casting content was least for all casting types. At $100 \%$ castings, sheep cast- ings had less air space than cattle or horse castings. As casting content increased, water holding capacity increased. Sheep castings generally had greater water holding capacity than cattle or horse castings. The commercial substrate control had the least bulk density at $0.08 \mathrm{~g} / \mathrm{cc}$ with a pore space of $90 \%$ and water holding capacity of $61 \%$. Percentage of air space in the commercial substrate was less than in the peat control. Sheep and cattle are ruminants and more completely digest cellulose than do horses. The large particles of undigested cellulose in the horse manure were difficult for the worms to eat and thus remained in the finished castings increasing the air space and decreasing water holding capacity. Sheep in the pasture will feed on shorter more tender grasses than cattle. It is likely the more tender grasses would digest more completely. When consumed by earthworms, the resulting castings were more dense and had greater bulk density than castings from cattle or horse. Values of total porosity and bulk density for peatmoss were similar to those reported by Fonteno (1993).

Within each casting type and at each measurement date, leachate $\mathrm{pH}$ increased as the casting content increased (Table 3 ). Castings are more basic than peatmoss and results from the basic nature of animal manure. When measured at week $2,4,6,8$, or 10 , substrates with high castings content remained more basic than the peatmoss control. At $100 \%$, castings from horse manure were consistently more acidic than those from cattle or sheep manure. The lower water holding capacity and greater undigested cellulose content of the castings from horse manure may contribute to the lower $\mathrm{pH}$ of the leachate. At $25 \%$ castings, there was no difference between the castings from cattle and horse manure. The $\mathrm{pH}$ of the Sunshine Mix 1 commercial substrate was similar to the $75 \%$ or $100 \%$ casting concentrations ranging from 6.1 at week 2 to 6.7 at week 10 .

Table 2. Physical properties of the treatment substrates composed of vermicompost (worm castings) from sheep, horse, or cattle manure and a 70 peat moss : 30 perlite mixture $(\mathrm{v} / \mathrm{v}) . \mathrm{BD}=0.08$, pore space $=90 \%$, air space $=30 \%$, and water holding capacity $=61 \%$ in Sunshine Mix 1 (SunGro Horticulture, Bellevue, Wash.), a commercial substrate used as an additional control.

\begin{tabular}{|c|c|c|c|c|c|c|c|c|c|c|c|c|}
\hline \multirow[b]{2}{*}{ Castings (\%) } & \multicolumn{3}{|c|}{ Bulk density $(\mathrm{g} / \mathrm{cc})$} & \multicolumn{3}{|c|}{ Pore space (\%) } & \multicolumn{3}{|c|}{ Air space (\%) } & \multicolumn{3}{|c|}{ Water holding capacity $(\%)$} \\
\hline & Sheep & Cattle & Horse & Sheep & Cattle & Horse & Sheep & Cattle & Horse & Sheep & Cattle & Horse \\
\hline$\overline{0}$ & $0.09 \mathrm{a}^{2}$ & $0.09 \mathrm{a}$ & $0.09 \mathrm{a}$ & $86 a$ & $86 a$ & $86 a$ & $36 a$ & $36 \mathrm{a}$ & $36 \mathrm{a}$ & $51 \mathrm{a}$ & $51 \mathrm{a}$ & $51 \mathrm{a}$ \\
\hline 25 & $0.13 \mathrm{~b}$ & $0.12 \mathrm{a}$ & $0.11 \mathrm{a}$ & $86 b$ & $84 a$ & $89 \mathrm{~b}$ & $31 b$ & 24 a & $32 b$ & $55 \mathrm{a}$ & $60 \mathrm{~b}$ & $57 \mathrm{a}$ \\
\hline 50 & $0.25 c$ & $0.13 \mathrm{a}$ & $0.14 b$ & $80 a$ & $86 b$ & $84 \mathrm{~b}$ & $16 \mathrm{a}$ & $25 \mathrm{~b}$ & $24 \mathrm{~b}$ & $63 \mathrm{~b}$ & $61 \mathrm{a}$ & $60 a$ \\
\hline 75 & $0.22 \mathrm{c}$ & $0.15 a$ & $0.17 \mathrm{~b}$ & $85 \mathrm{a}$ & $87 \mathrm{~b}$ & $85 \mathrm{a}$ & $19 a$ & $19 a$ & $26 \mathrm{~b}$ & $66 \mathrm{~b}$ & $68 \mathrm{~b}$ & 59 a \\
\hline 100 & $0.28 c$ & $0.16 \mathrm{a}$ & $0.17 \mathrm{~b}$ & $90 a$ & $93 \mathrm{~b}$ & $92 \mathrm{~b}$ & $12 \mathrm{a}$ & $24 \mathrm{~b}$ & $27 \mathrm{~b}$ & $77 \mathrm{c}$ & $69 \mathrm{~b}$ & $66 \mathrm{a}$ \\
\hline Significance & $\mathrm{L}^{* * *}$ & $\mathrm{~L}^{* * * *}$ & $L^{* * *}$ & $L^{* * \neq}$ & $\mathrm{L}^{* *+*}$ & $L^{\text {n*w }}$ & $\mathrm{L}^{* * *}$ & $\mathrm{~L}^{+* 4}$ & $\mathrm{~L}^{* * *}$ & $\mathrm{~L}^{* * *}$ & $\mathrm{~L}^{* * *}$ & $\mathbf{L}^{* * *}$ \\
\hline & $Q^{* * *}$ & $Q^{*}$ & $Q^{* * *}$ & $\mathrm{Q}^{* * *}$ & $\mathrm{Q}^{* * *}$ & $Q^{* * *}$ & $Q^{* * * *}$ & $\mathrm{Q}^{* * *}$ & $Q^{+* *}$ & $\mathrm{Q}^{* *}$ & $\mathrm{Q}^{* * * *}$ & $Q^{\text {ss }}$ \\
\hline
\end{tabular}

${ }^{2}$ Means separation using least significant differences at $P \leq 0.05$ within percentage of castings $(\mathrm{n}=6)$.

Ns. ******* Nonsignificant or significant at $P \leq 0.05,0.01$, or 0.001 . 
Table 3. Leachate pH measured every 2 weeks after planting until harvest of 'Miramar' chrysanthemum grown in vermicompost substrates. Substrates were composed of vermicompost (worm castings) from sheep, horse, or cattle manure and a 70 peatmoss : 30 pearlite mixture (v/v). Leachate pH was 6.1 at week 2,7.0 at week 4,6.1 at week 6,6.6 at week 8, and 6.7 at week 10 in Sunshine Mix 1 (SunGro Horticulture, Bellevue, Wash.), a commercial substrate used as an additional control.

\begin{tabular}{|c|c|c|c|c|c|c|c|c|c|c|c|c|c|c|c|}
\hline \multirow[b]{2}{*}{ Castings (\%) } & \multicolumn{3}{|c|}{ Week 2} & \multicolumn{3}{|c|}{ Week 4} & \multicolumn{3}{|c|}{ Week 6} & \multicolumn{3}{|c|}{ Week 8} & \multicolumn{3}{|c|}{ Week 10} \\
\hline & Sheep & Cattle & Horse & Sheep & Cattle & $\overline{\text { Horse }}$ & $\overline{\text { Sheep }}$ & Cattle & $\overline{\text { Horse }}$ & Sheep & Cattle & Horse & Sheep & Cattle & Horse \\
\hline 0 & $3.5 \mathrm{a}^{2}$ & $3.5 \mathrm{a}$ & $3.5 \mathrm{a}$ & $4.0 \mathrm{a}$ & $4.0 \mathrm{a}$ & $4.0 \mathrm{a}$ & $3.5 \mathrm{a}$ & $3.5 \mathrm{a}$ & $3.5 \mathrm{a}$ & $3.6 \mathrm{a}$ & $3.6 \mathrm{a}$ & $3.6 \mathrm{a}$ & $4.6 \mathrm{a}$ & $4.6 \mathrm{a}$ & $\overline{4.6 \mathrm{a}}$ \\
\hline 25 & $5.3 \mathrm{~b}$ & $4.5 \mathrm{a}$ & $4.6 \mathrm{a}$ & $5.8 \mathrm{~b}$ & $5.0 \mathrm{a}$ & $5.1 \mathrm{a}$ & $5.3 \mathrm{~b}$ & $4.4 \mathrm{a}$ & $4.3 \mathrm{a}$ & $5.4 \mathrm{~b}$ & $4.1 \mathrm{a}$ & $4.3 \mathrm{a}$ & $6.2 \mathrm{~b}$ & $5.5 \mathrm{a}$ & $5.5 \mathrm{a}$ \\
\hline 75 & $7.4 \mathrm{~b}$ & $7.5 \mathrm{~b}$ & $5.9 \mathrm{a}$ & $7.5 \mathrm{~b}$ & $7.6 \mathrm{~b}$ & $6.1 \mathrm{a}$ & $7.0 \mathrm{c}$ & $6.4 \mathrm{~b}$ & $5.1 \mathrm{a}$ & $6.7 \mathrm{~b}$ & $6.4 \mathrm{~b}$ & $4.8 \mathrm{a}$ & $6.4 \mathrm{~b}$ & $6.2 \mathrm{~b}$ & $5.5 \mathrm{a}$ \\
\hline 100 & $8.0 \mathrm{~b}$ & $8.1 \mathrm{~b}$ & $7.3 \mathrm{a}$ & $8.0 \mathrm{~b}$ & $7.9 \mathrm{~b}$ & $7.1 \mathrm{a}$ & $7.4 \mathrm{c}$ & $6.8 \mathrm{~b}$ & $5.8 \mathrm{a}$ & $6.9 \mathrm{~b}$ & $6.6 \mathrm{~b}$ & $5.6 \mathrm{a}$ & $6.7 \mathrm{~b}$ & $6.4 \mathrm{~b}$ & $5.9 \mathrm{a}$ \\
\hline Significance & $L^{* * *}$ & $\mathrm{~L}^{* * *}$ & $\mathrm{~L}^{* * *}$ & $L^{* * n}$ & $\mathrm{~L}^{* * *}$ & $\mathrm{~L}^{* * *}$ & $\mathrm{~L}^{* * *}$ & $L^{* * *}$ & $\mathrm{~L}^{* * *}$ & $\mathrm{~L}^{* * * *}$ & $\mathrm{~L}^{* * *}$ & $\mathrm{~L}^{* * *}$ & $L^{* * * *}$ & $\mathrm{~L}^{* * * x}$ & $L^{* * *}$ \\
\hline & $\mathrm{Q}^{* *}$ & $\mathrm{Q}^{* * *}$ & $Q^{\text {Ns }}$ & $\mathrm{Q}^{* * *}$ & $\mathrm{Q}^{* \star \star}$ & $\mathrm{Q}^{* * *}$ & $\mathrm{Q}^{* * *}$ & $\mathrm{Q}^{* * *}$ & $Q^{\text {ss }}$ & $\bar{Q}^{* * *}$ & $Q^{* s}$ & $Q^{\text {ss }}$ & $\mathrm{Q}^{* * *}$ & $\mathrm{Q}^{* *}$ & $\mathrm{Q}^{* *}$ \\
\hline
\end{tabular}

${ }^{2}$ Means separation using least significant differences at $P \leq 0.05$ within percentage of castings $(\mathrm{n}=8)$.

${ }_{* 5}, *, * *, * * *$ Nonsignificant or significant at $P \leq 0.05,0.01$, or 0.001 .

Table 4. Leachate electrical conductivity $\left(\mathrm{d} \cdot \mathrm{m}^{-1}\right)$ measured every 2 weeks after planting until harvest of 'Miramar' chrysanthemum grown in vermicompost substrates. Substrates were composed of vermicompost (worm castings) from sheep, horse, or cattle manure and a 70 peat moss : 30 perlite mixture (v/v). Electrical conductivity was 0.8 at week 2, 0.2 at week 4,1.9 at week 6,1.1 at week 8, and 1.3 at week 10 in Sunshine Mix 1 (SunGro Horticulture, Bellevue, Wash.), a commercial substrate used as an additional control.

\begin{tabular}{|c|c|c|c|c|c|c|c|c|c|c|c|c|c|c|c|}
\hline \multirow[b]{2}{*}{ Castings (\%) } & \multicolumn{3}{|c|}{ Week 2} & \multicolumn{3}{|c|}{ Week 4} & \multicolumn{3}{|c|}{ Week 6} & \multicolumn{3}{|c|}{ Week 8} & \multicolumn{3}{|c|}{ Week 10} \\
\hline & Sheep & Cattle & Horse & Sheep & Cattle & Horse & Sheep & Cattle & Horse & Sheep & Cattle & Horse & Sheep & Cattle & Horse \\
\hline$\overline{0}$ & $0.6 \mathrm{a}^{2}$ & $0.6 \mathrm{a}$ & $0.6 \mathrm{a}$ & $0.3 a$ & $0.3 \mathrm{a}$ & $0.3 \mathrm{a}$ & $1.6 \mathrm{a}$ & $1.6 \mathrm{a}$ & $1.6 \mathrm{a}$ & $1.3 \mathrm{a}$ & $1.3 \mathrm{a}$ & $1.3 \mathrm{a}$ & $1.4 \mathrm{a}$ & $1.4 \mathrm{a}$ & $\overline{1.4 \mathrm{a}}$ \\
\hline 25 & $2.7 \mathrm{~b}$ & $1.2 \mathrm{a}$ & $1.2 \mathrm{a}$ & $1.4 \mathrm{~b}$ & $0.4 \mathrm{a}$ & $0.4 \mathrm{a}$ & $2.6 \mathrm{~b}$ & $2.1 \mathrm{a}$ & $2.1 \mathrm{a}$ & $1.5 \mathrm{a}$ & $1.4 \mathrm{a}$ & $1.3 \mathrm{a}$ & $1.3 \mathrm{a}$ & $1.6 \mathrm{a}$ & $1.5 \mathrm{a}$ \\
\hline 75 & $2.3 \mathrm{c}$ & $1.0 \mathrm{a}$ & $1.5 \mathrm{~b}$ & $1.1 \mathrm{~b}$ & $0.7 \mathrm{a}$ & $0.8 \mathrm{ab}$ & $1.0 \mathrm{a}$ & $2.3 \mathrm{~b}$ & $1.8 \mathrm{~b}$ & $1.4 \mathrm{a}$ & $1.4 \mathrm{a}$ & $1.2 \mathrm{a}$ & $2.2 \mathrm{~b}$ & $1.4 \mathrm{a}$ & $1.7 \mathrm{a}$ \\
\hline 100 & $1.6 \mathrm{~b}$ & $1.2 \mathrm{a}$ & $1.1 \mathrm{a}$ & $0.9 \mathrm{ab}$ & $0.7 \mathrm{a}$ & $1.0 \mathrm{~b}$ & $1.2 \mathrm{a}$ & $1.9 \mathrm{~b}$ & $1.8 \mathrm{~b}$ & $1.1 \mathrm{a}$ & $1.3 \mathrm{a}$ & $1.1 \mathrm{a}$ & $1.4 \mathrm{a}$ & $1.5 \mathrm{a}$ & $1.0 \mathrm{a}$ \\
\hline \multirow[t]{2}{*}{ Significance } & $\mathrm{L}^{* * *}$ & $\mathrm{~L}^{* *}$ & $\mathrm{~L}^{* * *}$ & $\mathrm{~L}^{* *}$ & $\mathrm{~L}^{\mathrm{k*}}$ & $\mathrm{L}^{* * *}$ & $\mathrm{~L}^{\operatorname{nax}}$ & $\mathrm{L}^{\mathrm{vs}}$ & $\mathrm{L}^{\mathrm{vs}}$ & $\mathrm{L}^{\mathrm{Ni}}$ & $\mathrm{L}^{\text {ws }}$ & $\mathrm{L}^{\mathrm{Ns}}$ & $\mathrm{L}^{\mathrm{Ns}}$ & $L^{s s}$ & $\mathrm{~L}^{\mathrm{vs}}$ \\
\hline & $\mathrm{Q}^{* * * *}$ & $Q^{*}$ & $\mathrm{Q}^{* 4 \%}$ & $Q^{* * *}$ & $\mathrm{Q}^{\mathrm{ss}}$ & $Q^{\text {Ns }}$ & $\mathrm{Q}^{* * *}$ & $\mathrm{Q}^{* *}$ & $Q^{n s}$ & $Q^{\text {NS }}$ & $Q^{* s}$ & $Q^{\text {vs }}$ & $Q^{\text {ws }}$ & $Q^{\mathrm{Ns}}$ & $Q^{n s}$ \\
\hline
\end{tabular}

${ }^{2}$ Means separation using least significant differences at $P \leq 0.05$ within percentage of castings $(\mathrm{n}=8)$.

*s, *,**,**** Nonsignificant, or significant at $P \leq 0.05,0.01$ or 0.001 .

Leachate EC when measured at 2 weeks after planting increased as casting content increased to $50 \%$ or $75 \%$ before again decreasing in casings from sheep and horse respectively (Table 4). Leachate EC from cattle castings was greater than the control but increased little as the casting content increased. At 4 weeks, only the castings from sheep increased then decreased in leachate $\mathrm{EC}$ as castings concentration increased. The leachate $\mathrm{EC}$ from the cattle and horse castings increased linearly with concentration. After 8 weeks, regardless of casting concentration or type, there were no differences in leachate EC. At weeks 2 and 4, castings from sheep had greater leachate EC than cattle or horse at $25 \%$ to $100 \%$ castings. At week 6 , this trend reversed and castings from sheep had a reduced leachate EC compared to cattle and horse at $50 \%$ or greater castings. Leachate EC equalized between substrates over time due to initial high nutrient concentrations being either used by the plants or leached through regular irrigation events. The initial increase in leachate EC then decrease as castings concentration increased may be due to changes in porosity of the substrates as concentration of peatmoss decreased. Total porosity (Table 2 ) decreased before increasing as casting concentration increased. The leachate EC of the commercial substrate control was similar to the peatmoss perlite control at each week.

At 4 and 10 weeks after planting, the greatest plant growth was observed in substrates containing $50 \%$ sheep castings or $25 \%$ cattle or horse castings (Table 5). For all casting types, growth increased then decreased as castings concentration increased. When $25 \%$ casting was used there was no difference in growth between casting type 4 weeks after planting. However, at $50 \%$ casting, the largest plants were growing in sheep castings. When casting content was $25 \%$ or $50 \%$, the plants grew more in sheep castings after 10 weeks than in cattle or horse castings. Kiepas et al. (1998) also reported a negative effect on growth of tomato at $100 \%$ castings. At both 4 and 10 weeks after planting, plants in horse castings grew as large or larger than those in sheep castings at $75 \%$ or $100 \%$ castings. The greater growth in the horse castings at $75 \%$ or $100 \%$ castings may be attributed to the decreased water holding capacity of these substrates. A decreased water holding capacity increased the number of irrigations, and thus fertilizer, the plants received which may account for the greater growth. However, the largest plants overall were those grown in either $50 \%$ sheep castings or $25 \%$ cattle or horse castings. In addition, the substrate $\mathrm{pH}$ was lower in the horse than the cattle and sheep casting substrates at the $75 \%$ and $100 \%$ concentrations. The largest plants in sheep casting substrates were not associated with the lowest leachate $\mathrm{pH}$ at 10 weeks but the leachate $\mathrm{pH}$ was lower than 6.0 up until week 10 . The combination of a low $\mathrm{pH}$ and greater nutrient content of sheep castings may have resulted in the greater observed growth. As was also observed by Edwards and Burrows (1988), the substrates with castings had larger plants than the commercial control.

Foliar area and plant dry weight followed a pattern similar to growth index where foliar area and dry weight increased then decreased as casting concentration increased (Table 6). Greatest foliar area was on plants grown in $50 \%$ sheep or horse castings and $25 \%$ cattle castings. As with growth index, the greatest

Table 5. Growth index measured at 4 and 10 weeks after planting 'Miramar' chrysanthemum grown in vermicompost substrates. Plants were pinched 2 weeks after planting. Substrates were composed of vermicompost (worm castings) from sheep, horse, or cattle manure and a 70 peat moss : 30 perlite mixture $(\mathrm{v} / \mathrm{v})$. Growth index $=\pi \cdot\{[(\text { width } 1+\text { width } 2) / 2] / 2\}^{2} \cdot$ height. Growth index measured 2 weeks $\left(10938 \mathrm{~cm}^{3}\right)$ and 8 weeks $\left(18159 \mathrm{~cm}^{3}\right)$ after pinching plants grown in Sunshine Mix 1 (SunGro Horticulture, Bellevue, Wash.), a commercial substrate used as an additional control.

\begin{tabular}{|c|c|c|c|c|c|c|}
\hline \multirow[b]{3}{*}{ Castings (\%) } & \multicolumn{6}{|c|}{ Growth index $\left(\mathrm{cm}^{3}\right)$} \\
\hline & \multicolumn{3}{|c|}{ Week 2} & \multicolumn{3}{|c|}{ Week 8} \\
\hline & Sheep & Cattle & $\overline{\text { Horsc }}$ & Sheep & Cattle & Horse \\
\hline 0 & $7646 a^{2}$ & $7646 a$ & $7646 a$ & $10810 \mathrm{a}$ & $10810 \mathrm{a}$ & $\overline{10810 a}$ \\
\hline 25 & $13310 \mathrm{a}$ & $13137 \mathrm{a}$ & $12186 \mathrm{a}$ & $25752 \mathrm{~b}$ & $22330 a$ & $21571 \mathrm{a}$ \\
\hline 50 & $14044 b$ & $11085 \mathrm{a}$ & $11920 \mathrm{a}$ & $30439 \mathrm{~b}$ & $17105 \mathrm{a}$ & $17934 \mathrm{a}$ \\
\hline 75 & $9363 b$ & $7903 \mathrm{a}$ & $11775 \mathrm{c}$ & $15750 \mathrm{~b}$ & $12506 \mathrm{a}$ & $19259 \mathrm{c}$ \\
\hline 100 & $6211 b$ & $6090 \mathrm{a}$ & $7509 \mathrm{~b}$ & $12302 \mathrm{ab}$ & $9696 \mathrm{a}$ & $14416 \mathrm{~b}$ \\
\hline \multirow[t]{2}{*}{ Significance } & $\mathrm{L}^{* * * *}$ & $\mathrm{~L}^{\text {*4* } 4 \text { * }}$ & $\mathrm{L}^{\mathrm{N} s}$ & $L^{*}$ & $\mathrm{~L}^{* * * *}$ & $\mathrm{~L}^{\mathrm{vs}}$ \\
\hline & $\mathrm{Q}^{* * *}$ & $\mathrm{Q}^{n * *}$ & $\mathrm{Q}^{*+* *}$ & $Q^{*+* k}$ & $Q^{* * *}$ & $\mathrm{Q}^{* * *}$ \\
\hline
\end{tabular}

${ }^{2}$ Means separation using least significant differences at $P \leq 0.05$ within percentage of castings $(\mathrm{n}=8)$ ss, ***, *** Nonsignificant or significant at $P \leq 0.05,0.01$, or 0.001 . 
Table 6. Total foliar area, number of flowers per pot, number of days to flowering, plant dry weight, and depth of substrate (less equals more shrinkage) were measured at harvest of 'Miramar' chrysanthemum grown in vermicompost substrates. Substrates were composed of vermicompost (worm castings) from sheep, horse, or cattle manure and a 70 peatmoss : 30 perlite mixture $(\mathrm{v} / \mathrm{v})$. Total foliar area $\left(3268 \mathrm{~cm}^{2}\right)$, number of flowers per pot (157), number of days to flowering (55), plant dry weight $(63 \mathrm{~g})$, and depth of substrate $(10 \mathrm{~cm})$ measured on plants grown in Sunshine Mix 1 (SunGro Horticulture, Bellevue, Wash.), a commercial substrate used as an additional control.

\begin{tabular}{|c|c|c|c|c|c|c|c|c|c|c|c|c|c|c|c|}
\hline \multirow[b]{2}{*}{ Castings $(\%)$} & \multicolumn{3}{|c|}{ Foliar area $\left(\mathrm{cm}^{2}\right)$} & \multicolumn{3}{|c|}{ Dry wt (g) } & \multicolumn{3}{|c|}{ Flower number } & \multicolumn{3}{|c|}{ Days to flowering } & \multicolumn{3}{|c|}{ Substrate depth $(\mathrm{cm})$} \\
\hline & Sheep & Cattle & Horse & Sheep & Cattle & Horse & Sheep & Cattle & Horse & Sheep & Cattle & Horse & Sheep & Cattle & Horse \\
\hline$\overline{0}$ & $1870 a^{2}$ & $1870 \mathrm{a}$ & $1870 \mathrm{a}$ & $34 \mathrm{a}$ & $34 \mathrm{a}$ & $34 \mathrm{a}$ & $82 \mathrm{a}$ & $82 \mathrm{a}$ & $82 \mathrm{a}$ & $60 \mathrm{a}$ & $60 \mathrm{a}$ & $60 a$ & $10 \mathrm{a}$ & $10 \mathrm{a}$ & $10 \mathrm{a}$ \\
\hline 25 & $4102 \mathrm{~b}$ & $3365 \mathrm{a}$ & $3354 a$ & $74 \mathrm{~b}$ & $67 a$ & $67 \mathrm{a}$ & $188 \mathrm{~b}$ & $169 \mathrm{a}$ & $155 \mathrm{a}$ & $52 \mathrm{a}$ & $54 \mathrm{~b}$ & $53 \mathrm{ab}$ & $10 \mathrm{a}$ & $10 \mathrm{a}$ & $10 \mathrm{a}$ \\
\hline 75 & $2866 \mathrm{~b}$ & $2301 \mathrm{a}$ & $3152 \mathrm{c}$ & $58 \mathrm{~b}$ & $49 \mathrm{a}$ & $67 c$ & $160 \mathrm{ab}$ & $142 \mathrm{a}$ & $170 \mathrm{~b}$ & $52 \mathrm{a}$ & $56 \mathrm{~b}$ & $55 b$ & $9 a$ & $9 a$ & $9 a$ \\
\hline 100 & $2425 b$ & $2109 a$ & $2294 a b$ & $48 \mathrm{~b}$ & $43 \mathrm{a}$ & $55 \mathrm{c}$ & $141 \mathrm{~b}$ & $128 \mathrm{a}$ & $154 \mathrm{~b}$ & $54 \mathrm{a}$ & $56 \mathrm{~b}$ & $56 \mathrm{~b}$ & $8 \mathrm{a}$ & $8 \mathrm{a}$ & $8 a$ \\
\hline Significance & $L^{* s}$ & $L^{\mathrm{NS}}$ & $L^{\text {Ns }}$ & $\mathrm{L}^{* *}$ & $\mathrm{~L}^{\text {Ns }}$ & $L^{*+* *}$ & $L^{* * * *}$ & $\mathrm{~L}^{* * * *}$ & $\mathrm{~L}^{+* m}$ & $L^{*+*}$ & $\mathrm{~L}^{\mu * *}$ & $\mathrm{~L}^{* * *}$ & $\mathrm{~L}^{* * *}$ & $\mathrm{~L}^{* * *}$ & $\mathrm{~L}^{* * *}$ \\
\hline & $\mathrm{Q}^{* * * *}$ & $Q^{* \star *}$ & $\mathrm{Q}^{* * *}$ & $\mathrm{Q}^{* * *}$ & $\mathrm{Q}^{* * *}$ & $\mathrm{Q}^{* * *}$ & $\mathrm{Q}^{* * * *}$ & $Q^{\star \star *}$ & $\mathrm{Q}^{* * *}$ & $Q^{k+* *}$ & $\mathrm{Q}^{* * * *}$ & $\mathrm{Q}^{n+*}$ & $Q^{\text {**** }}$ & $Q^{* * * *}$ & $\mathrm{Q}^{* * *}$ \\
\hline
\end{tabular}

${ }^{2}$ Means separation using least significant differences at $P \leq 0.05$ within percentage of castings $(\mathrm{n}=8)$. ss. ${ }^{*}, * * * * *$ Nonsignificant or significant at $P \leq 0.05,0.01$, or 0.001 .

foliar area and dry weight were on plants in sheep castings when grown in up to $50 \%$ castings but were as great or greater in horse castings at $75 \%$ or $100 \%$ castings. The plants in commercial substrate had foliar area and dry weights similar to the plants in sheep castings at $75 \%$ and cattle and horse castings at 50\%. All plants in castings grew larger, had greater foliar area, and greater dry weight than plants grown in the peatmoss perlite control substrate. Dry matter of mung bean [Vigna radiata (L.) R. Wilcz. cv. K851] cultivated in cattle manure compost with earthworms was greater than in compost without earthworms (Sudha et al., 1999). The increased growth was attributed to greater nitrogen and phosphorus uptake when the earthworms were present in the compost.

Flower number peaked on plants grown in $50 \%$ sheep or cattle castings and $75 \%$ horse castings (Table 6). At $25 \%$ and $50 \%$ castings, flower number was greatest in sheep castings. However, flower number was as great or greater on plants in horse than sheep castings at $75 \%$ and $100 \%$ castings. All plants in castings or the commercial substrate had a greater number of flowers than the peatmoss perlite control.

Growing plants in the peatmoss perlite control delayed flowering by 4 to $7 \mathrm{~d}$ (Table 6). Within sheep casting substrates, plants in $100 \%$ castings required $2 \mathrm{~d}$ longer to flower. Flowering on plants grown in cattle castings occurred $54 \mathrm{~d}$ after pinching in $25 \%$ and $50 \%$ castings and was delayed 2 to $6 \mathrm{~d}$ when grown in $0 \%$, $75 \%$, or $100 \%$ castings. Plants grown in $25 \%$ horse castings flowered 2 to 7 d earlier than in any other horse casting concentration. At all casting concentrations, plants growing in sheep castings flowered earliest compared to those in cattle and horse castings. In the commercial substrate, the plants required $55 \mathrm{~d}$ from pinching to flower compared to the earliest flowering in sheep, cattle, and horse at 52,54, and $53 \mathrm{~d}$ respectively. The earliest flowering occurred in the same substrates that also produced the largest plants and greatest dry weights. Reduced time to flower has also been reported in chrysanthemum, salvia (Salvia splendens Sell ex Roem. \& Schult.), and petunia (Petunia $\times$ hybrida Hort. Vilm.-Andr.) grown in vermicompost and was attributed to a proposed presence of hormones in the vermicompost (Edwards and Burrows, 1988).

Substrate depth was measured at harvest to determine the amount of substrate shrinkage due to continued breakdown of the castings that may have occurred during plant growth. Depth remained constant at $10 \mathrm{~cm}$ until casting concentration reached $75 \%$ (Table 6). At $75 \%$ and $100 \%$, substrate depth was reduced to 9 and $8 \mathrm{~cm}$, respectively, for sheep, cattle and horse castings. Within casting concentration, there was no difference between casting type for substrate depth. This indicated that unless used at concentrations $>50 \%$, minimal substrate shrinkage occurred. In addition, it is an indication that the castings, after one month of vermicomposting, are relatively stable and exhibit minimal further decay over a 10 -week production cycle.

When growing chrysanthemums in soilless substrates, the general $\mathrm{pH}$ recommendation is between 5.5 to 6.0 (Ball, 1998). The $50 \%$ sheep casting substrate had $\mathrm{pH}$ values close to this range during the entire crop cycle. Also, during the first month after transplant, the $50 \%$ sheep casting substrate had the greatest EC and it has been shown that chrysanthemums require high amounts of $\mathrm{N}$ and $\mathrm{K}$ (Dole and Wilkins, 1999). Plants grown in mixtures of $25 \%$ or $50 \%$ sheep castings generally had greater growth index, dry weight, foliar area, number of flowers, and fewer days to flower than plants grown in any other substrate. A constant liquid feed with a 250 to $400 \mathrm{mg} \cdot \mathrm{L}^{-1}$ balanced fertilizer formula is recommended when growing chrysanthemums in soilless media. High levels of $\mathrm{N}$ during the first 7 weeks of the growth cycle are imperative. Deficiencies during this time will reduce the flower quality without chance of recovering (Larson, 1992). No further fertilization is required once the inflorescences reach a diameter of $\approx 1.25 \mathrm{~cm}$ as the nutrients required for the flowers during the last 3 weeks of the crop cycle will be translocated from the leaves (Larson, 1992). Even though the crop received a constant liquid feed at $300 \mathrm{mg} \cdot \mathrm{L}^{-1} \mathrm{~N}$, the additional nutrients provided from the sheep castings early in production proved beneficial and may have played an important role in the results. Handreck (1986) determined that vermicompost can be used successfully as a potting substrate for ornamental plant species since it provides nutrients required for at least a part of the plant cycle. The length of this time will depend on the plant species and on their nutritional requirement. Handreck (1986) also pointed out that when castings are present in the potting substrate it eliminated the need for addition of microelements and phosphorus, but nitrogen needed to be supplied for optimal plant growth.

It cannot be concluded, however, that casting nutrient content alone played the determinant role for better chrysanthemum plant performance. Plants grown in any of the mixtures of castings and peatmoss outperformed those grown in $100 \%$ castings and the peatmoss: perlite control. Casting substrates performed as well or better than the commercial substrate for all parameters measured.

Growth of chrysanthemum was positively affected by the addition of vermicompost to the substrate. Vermicompost produced from sheep manure outperformed that produced from cattle or horse manures. Substrates using vermicompost should be formulated with no more than $25 \%$ to $50 \%$ castings. Plants grown in $50 \%$ sheep or $25 \%$ cattle or horse casting substrates were of good marketable quality and were of greater size with a greater number of flowers and flowered earlier than plants grown in either the peatmoss perlite or commercial substrate controls.

\section{Literature Cited}

Alves, W. and A. Passoni. 1997. Compost and vermicompost of urban solid waste in Licania tomentosa (Benth) seedling production for arboriculture. Pesqui. Agropecu. Bras. 32:10531058 .

Ball, V. 1998. Ball red book. $16^{\text {th }}$ ed. Ball Publishing, Batavia, Ill.

Buchanan, M., G. Russell, and S. Block. 1988. Chemical characterization and nitrogen mineralization potential of vermicompost derived from differing organic wastes, p. 231-239. In: C. Edwards and E. Neuhauser (eds.). Earthworms in waste and environmental management. Academic, The Hague, The Netherlands.

Cox, M.S. 2001. The lancaster soil test method as an alternative to the Mehlich 3 soil test method. Soil Sci. 166:484-489.

Dole, J. and H. Wilkins, 1999. Floriculture, principles and species. Prentice Hall, Upper Saddle River, N.J.

Donald, D. and L. Visser. 1989. Vermicompost as a possible growth medium for the production of commercial forest nursery stock. Appl. Plant Sci. 3:110-113.

Edwards, C. 1988. Breakdown of animal, vegetable, and industrial organic wastes by earthworms, p. 21-23. In: C. Edwards and E. Neuhauser (eds.). 
Earthworms in waste and environmental management. Academic, The Hague, The Netherlands.

Edwards, C. and I. Burrows. 1988. The potential of earthworm compost as plant growth media, $p$. 211-219. In: C. Edwards and E. Neuhauser (eds.). Earthworms in waste and environmental management. Academic, The Hague, The Netherlands.

Fonteno, W. 1993. Problems and considerations in determining physical properties of horticultural substrates. Acta Hort. 324:197-204.

Handreck, K. 1986. Vermicomposts as components of potting media. Biocycle 27:58-62.

Hansen, M., H. Grønborg, N. Starkey, and L. Hansen. 1993. Alternative substrates for potted plants. Acta Hort. 342:191-196.

Kalembasa, S., J. Deska, and Z. Fiedorow. 1998. The possibility of utilizing vermicomposts in the cultivation of radish and paprika (in Polish).
Ann. Agr. Acad. Poznan 27:131-136.

Kiepas, K., M. Szczech, and Z. Fiedorow. 1998. Possibilities of using vermicompost from domestic wastes in ecological plant cultivation (in Polish). Ann. Agr. Acad. Poznan 27:137143.

Larson, R. 1992. Introduction to floriculture. $2^{\text {nd }} e d$. Academic, San Diego.

Miller, M. 2000. Double duty, double detail. Grower Talks 64:90-108.

Neal, K. 1991. Examine medium alternatives. Greenhouse Manager 10:42-48.

Orozco, F., J. Cegarra, L. Trujillo, and A. Roig. 1996. Vermicomposting of coffee pulp using the earthworm Eisenia fetida: Effects on $\mathrm{C}$ and $\mathrm{N}$ contents and the availability of nutrients. Biol. Fertil. Soils 22:162-166.

Riggle, D. and H. Holmes. 1994. Earthworms and composting: New horizons for commercial vermiculture. Biocycle 35(10):58-52.
Sanderson, K. and W. Martin. 1974. Performance of woody ornamentals in municipal compost medium under nine fertilizer regimes. HortScience 9:242-243.

Sudha, B., K. Kapoor, and S. Bansal. 1999. Effect of compost prepared from different farm wastes on growth and $\mathrm{N}$ and $\mathrm{P}$ uptake of mungbean. Environ. Ecol. 17:823-826.

Tomar, V., R. Bhatnagar, and R. Palta. 1998. Effect of vermicompost on production of brinjal and carrot. Indian Agr. Res. J. 13:153-156.

Wang, T. and F. Pokorny. 1989. Pecan shells as an organic component of container potting media. HortScience 24:75-78.

Wright, R. 1986. The pour through nutrient extraction procedure. HortScience 21:227-229.

Wright, R., K. Grueber, and C. Leda. 1990. Medium nutrient extraction with the pour-through and saturated medium extract procedures for poinsettia. HortScience 25:658-660. 